\title{
Interleukin-33 expression in ovarian cancer and its possible suppression of peritoneal carcinomatosis
}

\author{
ATSUSHI SEKIYA ${ }^{1}$, SHIRO SUZUKI ${ }^{1}$, AYAKO TANAKA ${ }^{1}$, SATOMI HATTORI ${ }^{1}$, YUSUKE SHIMIZU ${ }^{1}$, \\ NOBUHISA YOSHIKAWA ${ }^{1}$, YOSHIHIRO KOYA ${ }^{2}$, HIROAKI KAJIYAMA ${ }^{1}$ and FUMITAKA KIKKAWA ${ }^{1}$ \\ ${ }^{1}$ Department of Obstetrics and Gynecology, Nagoya University Graduate School of Medicine; \\ ${ }^{2}$ Bell Research Center for Reproductive Health and Cancer, Nagoya, Aichi 466-8550, Japan
}

Received January 24, 2019; Accepted July 1, 2019

DOI: $10.3892 /$ ijo.2019.4845

\begin{abstract}
Refractory peritoneal carcinomatosis is a common terminal feature of epithelial ovarian cancer (EOC). Previous reports have suggested that immunotherapy is a promising therapeutic strategy for EOC. Interleukin (IL)-33 is a member of the IL-1 superfamily of cytokines. The role of IL-33 in tissue inflammation and promoting type 2 immune responses has been established, and recently, there is accumulating evidence to suggest the involvement of IL-33 in carcinogenesis. In this study, we focused on the association between the tumor expression of IL-33 and ovarian peritoneal carcinomatosis. We used an immunosufficient murine model of peritoneal carcinomatosis and human EOC samples. The overexpression of IL-33 in the ID8 mouse EOC cell line tumors significantly prolonged the survival of immunocompetent mice in the peritoneal carcinomatosis setting, but not in the subcutaneous model. In addition, the silencing of IL-33 in ID8-T6 cells (subclone with high dissemination potential) significantly shortened the survival of the tumor-bearing mice. This was likely due to the intratumoral accumulation of $\mathrm{CD}^{+}$and $\mathrm{CD}^{+} \mathrm{T}$ cells, and a decrease in $\mathrm{CD}_{11} \mathrm{~b}^{+} \mathrm{Grl}^{+}$cells. Furthermore, IL-33 induced the intraperitoneal microenvironment favoring tumor elimination through the inhibition of differentiation into $\mathrm{CD} 1 \mathrm{~b}^{+} \mathrm{Gr} 1^{+}$cells. On the whole, the findings of this study suggest IL-33 to be a cytokine that reflects antitumor peritoneal conditions. Further investigation of the antitumorigenic role of IL-33 may aid in the development of more effective therapeutic approaches for the treatment of EOC with peritoneal carcinomatosis.
\end{abstract}

Correspondence to: Dr Shiro Suzuki, Department of Obstetrics and Gynecology, Nagoya University Graduate School of Medicine, 65 Tsuruma-cho, Showa-ku, Nagoya, Aichi 466-8550, Japan

E-mail: shiro-s@med.nagoya-u.ac.jp

Abbreviations: EOC, epithelial ovarian cancer; FCM, flow cytometry; IL, interleukin; IHC, immunohistochemical; MDSC, myeloid-derived suppressor cell; WB, western blot

Key words: ovarian cancer, peritoneal carcinomatosis, cytokine, interleukin-33, tumor-infiltrating lymphocyte

\section{Introduction}

Epithelial ovarian cancer (EOC) is the most lethal gynecological malignancy due to the fact that it is usually diagnosed at an advanced stage with peritoneal carcinomatosis in the majority of patients (1). Even with aggressive primary surgery and adjuvant chemotherapy, the majority of patients with advanced-stage EOC often suffer relapses and develop chemotherapeutic resistance (2). The recurrence and progression of peritoneal carcinomatosis leads to a poor survival and an impaired quality of life due to marked ascites. Therefore, novel treatment strategies are required to control peritoneal carcinomatosis.

Following the demonstration of EOC immunogenicity, multiple immunotherapeutic approaches remain underdeveloped (3-5). Immune checkpoint inhibitors, such as anti-cytotoxic T-lymphocyte-associated antigen 4 (CTLA-4) or anti-programmed death 1 (PD-1)/PD-L1 antibodies, have been considered as innovative treatments for a variety of malignancies. However, checkpoint inhibitors merely disinhibit ongoing T-cell responses. As a consequence, they often fail in tumors with a paucity of pre-existing tumor-infiltrating cytotoxic T lymphocytes.

Interleukin (IL)-1 family members are known to alter host responses to an infectious, inflammatory, or immunological challenge. IL-33 is a member of the IL-1 family that has been identified as a potent activator of the immune system. IL-33 is released by damaged cells, acting as an alarmin (6). IL-33 functions by interacting with its receptors, ST2 (also known as IL1RL1) and IL-1RAcP $(7,8)$. IL-33 has been reported to be associated with a number of diseases, including infections $(9,10)$, asthma $(11,12)$, autoimmune diseases $(13,14)$, atherosclerosis and cardiovascular disease $(15,16)$. However, there are only a few reports available to date on the role of IL-33 signaling in cancer. Recently, the high expression of IL-33 and its receptor, ST2, were reported to be poor prognostic factors for survival. IL-33 was shown to promote gastric cancer cell migration and invasion by stimulating the secretion of IL-6 and MMP-3 (17). Increased IL-33 protein levels have been observed in serum and liver tissue from patients with hepatocellular carcinoma (18). Furthermore, IL-33 and ST2 expression levels have been shown to be higher in human breast cancer tissue than in normal breast tissue $(19,20)$. By contrast, a protective role for IL-33 has been reported in other studies. 
The IL-33/ST2 signaling axis may play a protective role in colon carcinogenesis through macrophage infiltration (21), and IL-33 may increase the death of ST2-expressing lung cancer cells under conditions mimicking the tumor environment (22). The reports differ depending on the type of cancer; therefore, the function of IL-33 is controversial.

In ovarian cancer, a high expression of IL-33 has been reported to be associated with a poor prognosis of patients with EOC $(23,24)$; however, to date, at least to the best of our knowledge, the association between ovarian cancer and the function of IL-33 has not yet been fully elucidated. We investigated the correlation between IL-33 and EOC in the peritoneal carcinomatosis environment, which is likely to be accessible and refractory in advanced EOC.

\section{Materials and methods}

Cells and cell culture. Murine ovarian cancer cell lines (ID8 and its subclones ID8-T6, ID8-mock and ID8-IL-33) and human EOC cell lines (SKOV-3/CAOV3/OV90/A2780) were used in this study. The ID8 cell line was kindly provided by Dr Katherine Roby (University of Kansas Medical Center). The SKOV-3, CAOV3 and OV90 cell lines were obtained from the American Type Culture Collection (ATCC). The A2780 cell line was obtained from the European Collection of Cell Cultures (ECACC). These cells were maintained in RPMI-1640 medium (Sigma-Aldrich) supplemented with $10 \%$ fetal calf serum, penicillin $(100 \mathrm{U} / \mathrm{ml})$ and streptomycin $(100 \mathrm{~g} / \mathrm{ml})$ at $37^{\circ} \mathrm{C}$ in a humidified atmosphere containing $5 \% \mathrm{CO}_{2}$. All cell lines were regularly tested for mycoplasma contamination.

Animal and tumor model. A total of 95 C57BL/6 female mice (89 4-5 weeks old mice; weight range, 13.8-18.0 g; mean weight, $15.6 \mathrm{~g}$ and 6 10-12 weeks old mice) and $8 \mathrm{BALB} / \mathrm{C}$ nude female mice (5 weeks old; weight range, 16.8-18.6 g; mean weight, $18.0 \mathrm{~g}$ ) were purchased from Charles River Laboratories Japan. Animal care and experimental procedures were approved by the Animal Experiment Committee of Nagoya University (approval no. 30065), and all animals were maintained under specific pathogen-free conditions. Mice were housed in groups of 3 or 4 in cages and kept in a room maintained at $23 \pm 1^{\circ} \mathrm{C}$ with free access to food and water, on a 12-h light-dark cycle throughout the experiments. The mice were allowed to acclimatize to their conditions for 2 weeks. The C57BL/6 mice and BALB/C nude mice were intra-abdominally injected with $5.0 \times 10^{6}$ tumor cells in $0.3 \mathrm{ml}$ of PBS to induce peritoneal metastasis. Mice with peritoneal carcinomatosis with a body weight $>25 \mathrm{~g}$ were sacrificed, and intraperitoneal dissemination was evaluated. We generated the subclone of ID8 with higher peritoneal dissemination capacity. Peritoneal dissemination was harvested and cultured in medium, and tumor cells were re-implanted intraperitoneally into 3 different C57BL/6 mice. This re-implantation was performed a total of 5 times to establish the ID8-T6 mice (Fig. S1). We used $18 \mathrm{C} 57 \mathrm{BL} / 6$ mice in this process (3 mice for initial disseminated tumor formation and we repeated the re-implantation of 3 mice each 5 times).

The C57BL/6 mice were injected with 5.0x10 6 tumor cells in $0.2 \mathrm{ml}$ of PBS into the right rear flank of each mouse to form subcutaneous tumors. Subcutaneously inoculated mice were sacrificed at 6 weeks following the injection. Subcutaneous tumor size was measured weekly thereafter using a digital Vernier caliper. Tumor volumes were calculated using the following formula: $\mathrm{V}=\left(\right.$ longest diameter $\mathrm{x}$ shortest diameter $\left.{ }^{2}\right) / 2$.

Humane endpoints. Humane euthanasia using $\mathrm{CO}_{2}$ was performed when a mouse reached an experimental endpoint, was sampled with irreversible and persistent pain or became moribund. The flow rate and container volume for $\mathrm{CO}_{2}$ asphyxiation were $2.0 \mathrm{l} / \mathrm{min}$ and container volume was $10 \mathrm{l}$, respectively. To confirm mouse death, we checked for the absence of multiple vital signs (loss of bladder control, absence of heart rate, lack of a toe-pinch response and cessation of respiration). In order to obtain a rapid and sufficient amount of bone marrow cells, they were collected following euthanasia. To minimize animal suffering and distress at the time of invasive procedures, the mice were anesthetized by isofluorane inhalation (2-5\%) administered by nose cone to reach a steady state of anesthesia, which was determined by toe pinch reflex and slow steady breathing.

Vectors andtransductions.pSIREN-RetroQ-ZsGreen-Control (5'-TTCTCCGAACGTGTCACGT-3') and -mIL33-2334 (5'-AGGTATAATTGTTTCATTAATTT-3') are small hairpin RNA (shRNA) expression vectors encoding non-target shRNA or shRNA against mouse IL-33 (IL33-2334), respectively. pRetroX-IRES-DsRedExpress-mIL33 is a mammalian expression vectorencoding mouse IL-33.pRetroX-IRES-DsRedExpress-Mock, which is an empty vector, was used as a control.

The ID8-T6 cells were transduced with pSIRENRetroQ-ZsGreen-Control and -mIL33-2334, which were used for gene silencing. The ID8 cells were transduced with pRetroX-IRES-DsRedExpress-Mock and -mIL33, which were used for gene overexpression. The vector transfections were carried out using Lipofectamine 3000 (Thermo Fisher Scientific). To produce viral for delivery, 293T cells were transfected with pSIREN-RetroQ-DsRed-Express (or pRetroX-IRES-DsRed Express), VSV and gag-pol. After 3 days, the supernatant was collected and filtrated $(0.45 \mu \mathrm{m})$. Filtrated supernatant with polybrene (final $5 \mathrm{mg} / \mathrm{ml}$ ) was added to ID8-T6 (or ID8) cells and incubated for $24 \mathrm{~h}$ at $37^{\circ} \mathrm{C}$.

Both pRetroX-IRES-DsRed Express and pSIREN-RetroQZsGreen were purchased from Clontech (Takara Bio). All oligo DNA were synthesized by and purchased from Hokkaido System Science.

Immunohistochemical (IHC) staining. IHC analyses were performed on human EOC tissues and intraperitoneally transplanted tumors from mice. The human EOC tissues were obtained from 100 patients who underwent surgical treatment at Nagoya University Hospital between 1989 and 2011. All samples were fixed in 10\% formalin and embedded in paraffin. The sections were cut at a thickness of $5 \mu \mathrm{m}$. For heat-induced epitope retrieval, deparaffinized sections in $0.1 \mathrm{M}$ citrate buffer were treated at $90^{\circ} \mathrm{C}$ at $750 \mathrm{~W}$ for 15 min using a microwave oven. Immunostaining was conducted using Histofine Simple Stain MAX PO (MULTI) (424151, Nichirei Biosciences), followed by incubation at $4^{\circ} \mathrm{C}$ overnight with the primary antibodies. Histofine Simple Stain MAX PO is the labeled 
polymer prepared by combining amino acid polymers with peroxidase and secondary antibody which is reduced to Fab' fragment. We applied 2 drops of this detection reagent to each slide so as to provide a complete cover of the sections. This was followed by incubation at room temperature for $30 \mathrm{~min}$. For IL-33, CD4 and CD8, the samples were incubated with rabbit anti-IL-33 (clone Nessy-1, 1:1,000 dilution; ALX-804-840, Enzo Life Sciences), rabbit anti-CD4 (clone SP35, 1:100 dilution; M3354, Spring Bioscience) and mouse anti-CD8 (clone C8/144B, 1:100 dilution; M7103, Dako).

As regards the IHC staining of mouse tumors, tissues removed from the mice were immersed in OCT compound (Sakura Finetek) and rapidly frozen and stored at $-80^{\circ} \mathrm{C}$. Frozen tissue sections were cut at $5 \mu \mathrm{m}$ thickness using a cryostat microtome at $-15^{\circ} \mathrm{C}$, air-dried and fixed in acetone for $10 \mathrm{~min}$. The sections were then incubated with rat anti-CD4 (clone GK1.5, 1:100 dilution; 100401, BioLegend), rat anti-CD8a (clone 53-6.7, 1:100 dilution; 100701, BioLegend), rat anti-CD11b (clone M1/70, 1:500 dilution; 101201, BioLegend) and rat anti-F4/80 (clone BM8, 1:500 dilution; 123101, BioLegend) at room temperature overnight. The sections were then washed 3 times in PBS, and 2 drops of Histofine Simple Stain Mouse MAX-PO (Rat) (414311, Nichirei Biosciences) reagent were added for $30 \mathrm{~min}$ at room temperature.

Immunohistochemical evaluation. The samples were classified according to the intensity of IL-33 staining and scored from 0 to 3 as follows: 0 , negative; 1 , weak; 2 , moderate; and 3 , strong. Cases with scores of 0 and 1 belonged to the IL-33 low group and cases with scores of 2 or 3 belonged to the IL-33 high group. Tumor-infiltrating $\mathrm{CD}^{+}$and $\mathrm{CD}^{+}$cells were counted at $\mathrm{x} 400$ magnification, $\mathrm{CD} 1 \mathrm{~b}^{+}$and $\mathrm{F} 4 / 80^{+}$cells were counted at $\times 200$ magnification in 4 different microscopic fields, and the average number was calculated (using a ZEISS Axio Imager A1 microscope). The scoring of IL-33 intensity and counting of tumor-infiltrating immune cells were carried out twice by two independent gynecologists (each blinded to the other's score) without any knowledge of the patient clinical parameters or other prognostic factors. The concordance rate was $>90 \%$ between the observers.

Flow cytometric analysis. Flow cytometry (FCM) was carried out to quantify the expression of CD11b and Gr-1 on the surface of the cells. Mice with tumor formation were sacrificed by $\mathrm{CO}_{2}$ gas asphyxiation and their disseminated tumors were rapidly collected. The cells were stained with the following antibodies for $30 \mathrm{~min}$ at $4^{\circ} \mathrm{C}$ : APC anti-mouse CD11b (clone M1/70; 101201, BioLegend) and PE anti-mouse Gr-1 (clone RB6-8C5; 108407, BioLegend). FCM data were acquired using the Attune Acoustic Focusing Cytometer (Life Technologies; Thermo Fisher Scientific), and analyzed using Attune cytometric software version 2.1.0. Gating was implemented on the basis of negative-control staining profiles.

RNA isolation and gene expression analysis. Total RNA was isolated from the cells and tissues using the RNeasy RNA Isolation kit (Qiagen) and cDNA was synthesized using the ReverTraAce qPCR RT kit (Toyobo). The approximate size of the PCR products was estimated by the electrophoresis of $5 \mu 1$ of each PCR reaction on 1.0\% agarose gel containing GelRed
(Biotium). Real-time PCR was performed on a LightCycler using SYBR-Green 1 Master (Roche). The $2^{-\Delta \Delta \mathrm{Cq}}$ method was adopted for quantification (25). The primary PCR primer pairs are listed in Table SI. Microarray analyses were performed using the Affymetrix GeneChip. The reaction was performed for $10 \mathrm{~min}$ at $30^{\circ} \mathrm{C}, 20 \mathrm{~min}$ at $42^{\circ} \mathrm{C}$ and $5 \mathrm{~min}$ at $99^{\circ} \mathrm{C}$, and then stored at $4^{\circ} \mathrm{C}$.

Western blot (WB) analysis. WB analysis for IL-33 and receptor ST2 was performed as previously described (26). In brief, cells were treated with 10\% RIPA lysis buffer (Thermo Fisher Scientific) in PBS and cOmplete, Mini, EDTA-free (Roche). Subsequently, $30 \mu \mathrm{g}$ of total cell lysate were electrophoresed on a $10 \%$ SDS-polyacrylamide gel and transferred electrophoretically to Immobilon membranes (Millipore). After blocking in blocking solution (5\% non-fat dry milk/0.1\% Tween-20/PBS) at room temperature for $1 \mathrm{~h}$, the membranes were incubated overnight with a recommended dilution of primary antibodies. The following antibodies were used at $4^{\circ} \mathrm{C}$ overnight: Anti-mouse and human IL-33 (clone Nessy-1; 1:1,000 dilution; ALX-804-840, Enzo Life Sciences), anti-human ST2 (1:1,000 dilution; PRS3363-100UG, Sigma-Aldrich) and anti- $\beta$-actin (1:5,000 dilution; 017-24573, Wako). The primary antibodies were washed in $0.05 \%$ Tween-20/PBS and then incubated with the appropriate HRP-linked secondary antibody at room temperature for $1 \mathrm{~h}(1: 10,000 ; 7074$ and 7076, Cell Signaling Technology). Proteins were detected with an ECL kit (GE Healthcare Life Science) and visualized using the ImageQuant LAS 4000 Mini system (GE Healthcare Life Science).

Determination of the IL-33 concentration. The IL-33 concentration in the supernatant and ascites was evaluated by ELISA (murine IL-33; R\&D Systems). The ID8-WT, ID8-T6, ID8-mock and ID8-IL-33 cells ( $5 \times 10^{5}$ cells/well) were seeded in 6-well plates and incubated in appropriate culture medium for $24 \mathrm{~h}$ at $37^{\circ} \mathrm{C}$. After reaching confluence, the cells were washed with serum-free medium, and incubated for a further $48 \mathrm{~h}$ at $37^{\circ} \mathrm{C}$. Following incubation, the supernatants were collected for the assay. Ascites were harvested from ID8-mock or ID8-IL-33 peritoneal tumor-bearing mice and the cells were removed by centrifugation $\left(500 \times \mathrm{g}, 5 \mathrm{~min}\right.$ at $\left.4^{\circ} \mathrm{C}\right)$ for the assays.

In vitro cell proliferation assay. The cells were plated in hexaplicate at a density of 1,000 cells in $200 \mu 1$ in 96-well plates, and cultured for 1 to 3 days at $37^{\circ} \mathrm{C}$. Cell viability was assayed using a modified tetrazolium salt MTT assay performed using the CellTiter 96 Aqueous One Solution Cell Proliferation Assay kit (Promega). The absorbance was measured at $490 \mathrm{~nm}$ using a microplate reader (Labsystems, Multiskan Bichromatic).

Myeloid-derived cell generation assay. Bone marrow cells were harvested from the femurs of 6 healthy female C57BL/6 mice (10-12 weeks old). The bone marrow cells were cultured in RPMI-1640 with 10\% FBS in the presence of ascites fluid that was extracted from ID8-mock or ID8-IL-33 peritoneal tumor-bearing mice from which the cells were removed by centrifugation $\left(500 \mathrm{x} \mathrm{g}, 5 \mathrm{~min}\right.$ at $\left.4^{\circ} \mathrm{C}\right)$. The cells were collected on day 7 for FCM. For stimulation with IL-33, recombinant 
Table I. Association between the expression of IL-33 and the clinicopathological parameters of the patients with EOC.

\begin{tabular}{|c|c|c|c|c|}
\hline \multirow[b]{2}{*}{ Parameter } & \multirow[b]{2}{*}{ No. } & \multicolumn{2}{|c|}{ IL-33 expression } & \multirow[b]{2}{*}{ P-value } \\
\hline & & Low, n (\%) & High, n (\%) & \\
\hline Total & 100 & 33 & 67 & \\
\hline Age (years) & & & & 0.288 \\
\hline$\leq 55$ & 50 & $14(42.4)$ & $36(53.7)$ & \\
\hline$>55$ & 50 & $19(57.6)$ & $31(46.3)$ & \\
\hline FIGO stage & & & & 0.792 \\
\hline I & 31 & $12(36.4)$ & $19(28.4)$ & \\
\hline II & 20 & $5(15.2)$ & $15(22.4)$ & \\
\hline III & 43 & $14(42.4)$ & $29(43.3)$ & \\
\hline IV & 23 & $2(6.1)$ & $4 \quad(6.0)$ & \\
\hline Histological type & & & & 0.664 \\
\hline Serous & 48 & $16(48.5)$ & $32(47.8)$ & \\
\hline Clear & 29 & $11(33.3)$ & $18(26.9)$ & \\
\hline Endometrioid & 23 & $6(18.2)$ & $17(25.4)$ & \\
\hline Grade & & & & 0.205 \\
\hline G1 & 8 & $4(12.1)$ & $4 \quad(6.0)$ & \\
\hline $\mathrm{G} 2$ & 31 & $6(18.2)$ & $25(37.3)$ & \\
\hline G3 & 57 & $22(66.7)$ & $35(52.2)$ & \\
\hline Unknown & 4 & $1(3.0)$ & $3(4.5)$ & \\
\hline CA125 (U/ml) & & & & 0.229 \\
\hline$<350$ & 49 & $19(57.6)$ & $30(44.8)$ & \\
\hline$\geq 350$ & 51 & $14(42.4)$ & $37(55.2)$ & \\
\hline
\end{tabular}

mouse IL-33 (R\&D Systems) at 20, 200 or 2,000 was added to each well on day 1.

Scratch wound migration assay (wound healing assay). Tumor cells were seeded on 6-well plates and incubated at $37^{\circ} \mathrm{C}$ overnight. The cell monolayer was then scratched using a 200- $\mu$ l pipette tip and washed twice with PBS to ensure the scratch area. The cells were examined $8 \mathrm{~h}$ after the scratch was made using an OLYMPUS IX71 microscope (Olympus).

Statistical analysis. For the results of in vitro and in vivo experiments, statistical comparisons between groups were performed using the non-paired Student's t-test. The comparisons between multiple groups were assessed using one-way ANOVA, followed by Tukey's test. Overall survival curves were generated using the Kaplan-Meier method and compared using a log-rank test. The comparison between the IL-33 expression levels in different groups was assessed using Chi-squared tests (Table I). Differences between groups were considered significant at $\mathrm{P}<0.05$. Data are expressed as the means $\pm \mathrm{SD}$.

\section{Results}

Generation and characterization of invasive ID8-T6 ovarian tumor cells. The ID8 murine ovarian cancer cell line slowly forms tumors when injected intraperitoneally in C57BL/6 mice. In order to investigate the mechanisms of peritoneal dissemination, we prepared a more invasive subclone of ID8. A syngeneic mouse model was established by injecting ID8 cells intraperitoneally into C57BL/6 mice. Peritoneal dissemination was harvested and cultured in medium, and tumor cells were re-implanted intraperitoneally into different C57BL/6 mice. This re-implantation was repeated 5 more times to create the invasive ID8 cell line ID8-T6 (Fig. S1).

The ID8-WT and ID8-T6 cell lines were intraperitoneally injected into C57BL/6 mice, and their survival was compared. ID8-T6 caused the earlier accumulation of ascites and the formation of dissemination compared with ID8-WT (Fig. S2A). As shown in Fig. S2B, the ID8-T6 tumor-bearing mice had a significantly poorer survival than the ID8-WT tumor-bearing mice $(\mathrm{P}=0.002)$. Based on the above, ID8-T6 became a cell line that more easily formed peritoneal dissemination. In order to confirm whether there was a difference between ID8-WT and ID8-T6 in vitro, cell proliferation was examined. No marked differences were observed in the growth rates between the ID8-WT cells and ID8-T6 cells (Fig. S2C). We also examined the effects of ID8-T6 on cell migration by wound healing assay, in which cells migrate from the edge of a scratch wound. ID8-T6 cells had spread along the wound edges more rapidly than the ID8-WT cells at $8 \mathrm{~h}$ (Fig. S3).

To identify which genes promote peritoneal carcinomatosis, we performed microarray analysis to compare the ID8-WT cells with ID8-T6 cells (in vitro conditions) and ID8-WT tumor tissue with ID8-T6 tumor tissue (in vivo conditions). Among the genes listed, we focused on humoral factors. IL-33 was 
A

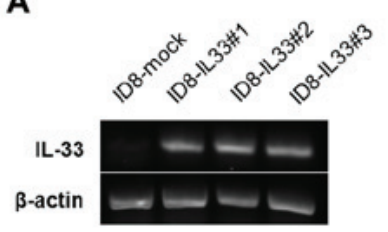

B

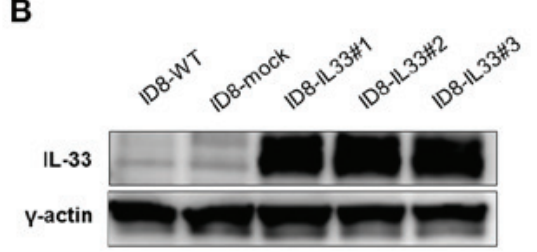

C

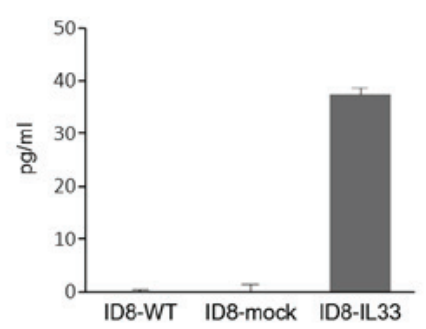

D

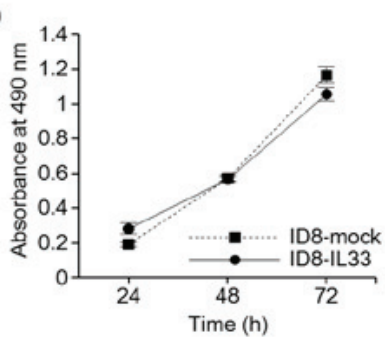

G
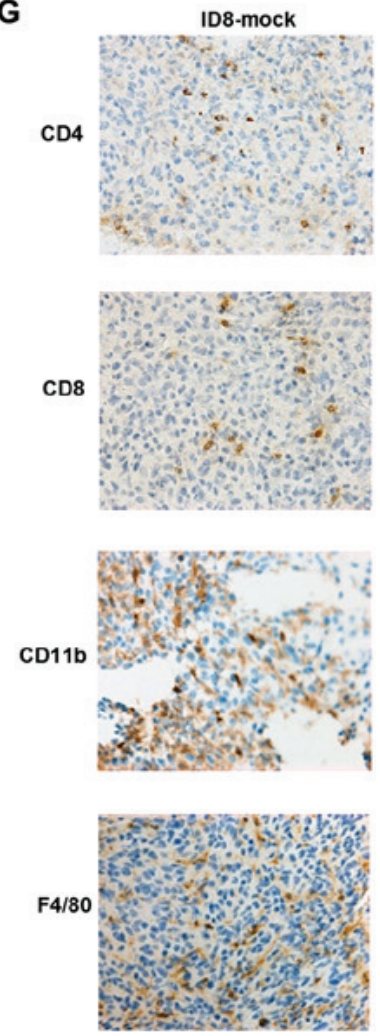

E

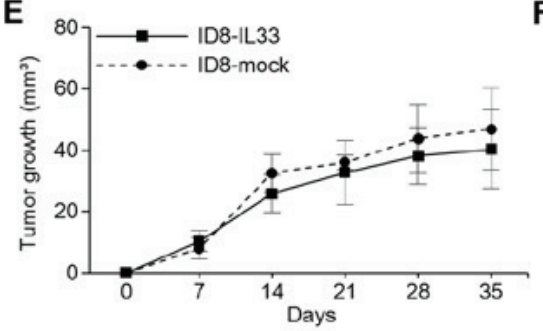

$\mathbf{F}$

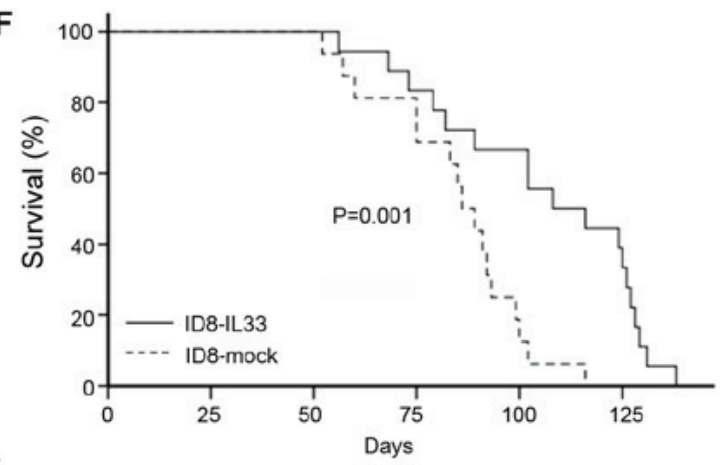

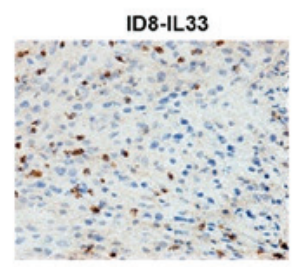
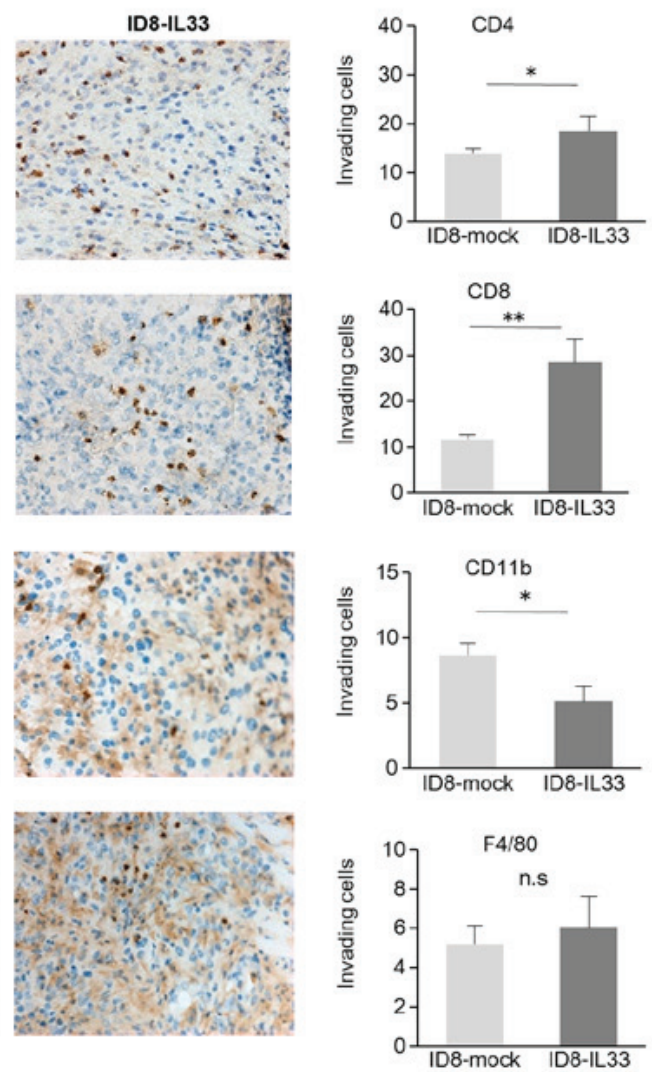

Figure 1. IL-33 increases the number of tumor-infiltrating $\mathrm{CD}^{+}$and $\mathrm{CD} 8^{+}$cells, but decreases $\mathrm{CD} 11 \mathrm{~b}^{+}$cells. (A) Semi-quantitative PCR analysis of IL-33 expression in ID8-mock and ID8-IL33 cells. (B) Western blot analysis of IL-33 expression in ID8-mock and ID8-IL33 cells. (C) Concentration of IL-33 in cell culture supernatant of ID8-mock or ID8-IL33 cells. (D) Cell proliferation was measured over 3 days for ID8-mock and ID8-IL33 cells using the MTT assay. No difference in proliferation was observed. Data are presented as absorbance at optical density (OD) of $490 \mathrm{~nm}$. (E) Subcutaneous tumor growth (mm ${ }^{3}$ ) in C57BL/6 mice after injection of ID8-mock or ID8-IL33 cells $\left(5 \times 10^{6}\right)$ into the right rear flank of each mouse. One tumor was removed per mouse. Mean $( \pm$ SEM) tumor growth over time in mice ( $\mathrm{n}=4$ for each group). (F) Overall survival rates were compared between mice intraperitoneally injected with ID8-mock ( $\mathrm{n}=16)$ or ID8-IL33 $(\mathrm{n}=18)$ cells $\left(1 \times 10^{7}\right) \mathrm{P}=0.001$. (G) Representative immunostaining images of ID8-mock or ID8-IL33 tumors for CD4, CD8, $\mathrm{CD} 11 \mathrm{~b}$ and $\mathrm{F} 4 / 80$. Analyses of the infiltration of $\mathrm{CD}^{+}, \mathrm{CD}^{+}, \mathrm{CD} 11 \mathrm{~b}^{+}$and $\mathrm{F} 4 / 80^{+}$cells within tumors $(\mathrm{n}=4) .{ }^{*} \mathrm{P}<0.05$ and ${ }^{* * *} \mathrm{P}<0.001$.

found to be upregulated in both the ID8-T6 cells and tissue as compared with ID8-WT (15.1-fold higher, 50.7-fold higher, Table SII). To confirm these results, we performed RT-qPCR on the ID8-WT and ID8-T6 cells for 10 genes [IL-6, IL-8, IL-13, IL-33, interleukin 1 receptor like 1 (IL1RL1), transforming growth factor (TGF)- $\beta 1$, heparin binding EGF like growth factor (HB-EGF), vascular endothelial growth factor (VEGF) $\alpha, \mathrm{C}-\mathrm{C}$ motif chemokine ligand 5 (CCL5) and prostaglandin $\mathrm{E}$ receptor 2 (PTGER2)]. Significant differences were found for all genes. IL-33 and its receptor IL1RL1 were significantly upregulated (Fig. S2D). In addition, the increased protein expression of IL-33 was observed by WB and IHC analyses (Fig. S2E and F). Furthermore, we confirmed the expression of IL-33 in human ovarian cancer. We also examined the expression of IL-33 and its receptor ST2 in human ovarian cancer cell lines (SKOV3, CAOV3, OV90 and A2780) by WB 
A
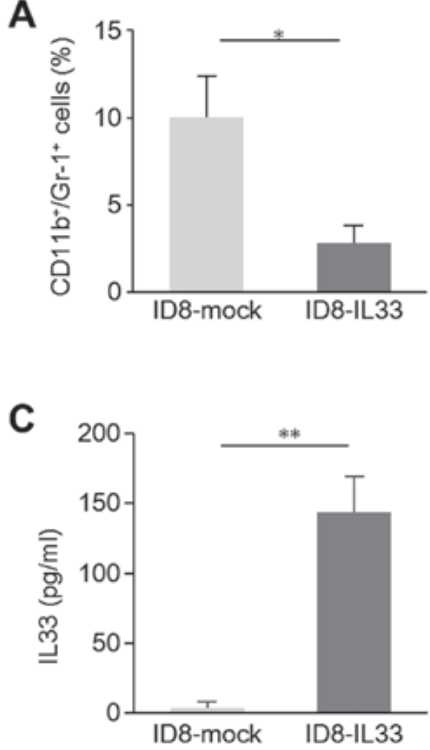

B

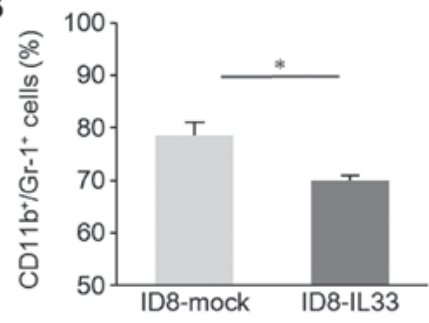

D

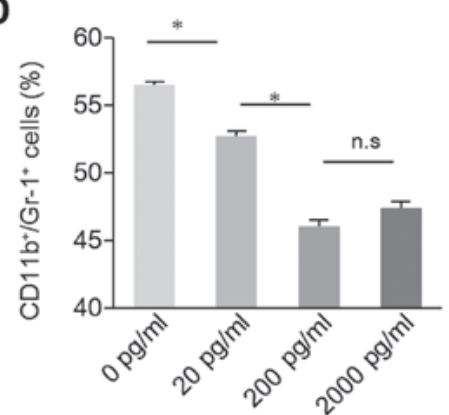

Figure 2. IL-33 in ascites inhibits the differentiation of bone marrow cells into MDSCs. (A) Flow cytometric analysis of the accumulation of CD11b ${ }^{+}$Gr1 ${ }^{+}$cells (MDSCs) in tumor cells. (B) Flow cytometric analysis of the frequencies of bone-marrow-derived MDSCs. Ascites from ID8-mock or ID8-IL33 tumor-bearing mice was added to the culture medium. (C) The concentration of IL-33 in ascites of ID8-mock or ID8-IL33 tumor-bearing mice. (D) Frequencies of bone-marrow-derived MDSCs by flow cytometry. Recombinant IL-33 (20, 200 or 2,000 pg/ml) was added to the culture medium. * $\mathrm{P}<0.05$ and ${ }^{* *} \mathrm{P}<0.001$; n.s, not significant. MDSCs, myeloid-derived suppressor cells.

analysis. No expression of IL-33 was observed in the A2780 cell line; however, both IL-33 and ST2 were found to be expressed in the SKOV3, CAOV3 and OV90 cell lines (Fig. S4). This finding suggested that IL-33 was highly expressed in ID8-T6 cells and may thus be involved in human ovarian cancer.

In vitro functional experiments on ID8-IL33 ovarian tumor cells. To examine the effects of IL-33 on peritoneal carcinomatosis, ID8-WT, a cell line lacking IL-33, was gene-transferred with IL-33 cDNA, and ID8-IL33 cells expressing IL-33 and ID8-mock control cells were generated. The expression of IL-33 following gene transfer was confirmed by semi-quantitative PCR and WB analysis (Fig. 1A and B). In addition, the IL-33 concentrations in the cell culture supernatant were examined. It was confirmed that the IL-33 concentration in the ID8-IL33 cells was high enough (Fig. 1C). We then further examined whether there was a difference between the ID8-IL33 and ID8-mock cells in vitro by evaluating cell proliferation. However, the in vitro growth rates between the ID8-IL33 cells and ID8-mock cells did not differ (Fig. 1D). We also assessed whether IL-33 promotes the migratory ability of the tumor cells by scratch wound migration assay; however, there was no difference in the migratory ability between the ID8-IL33 and IL33-mock cells (Fig. S5). Thus, the overexpression of IL-33 did not affect tumor cell proliferation or migration in vitro.

IL-33 does not promote tumor progression, but increases tumor immunity by promoting the infiltration of immune cells into the tumor. As IL-33 had no effect on tumor cells in vitro, we decided to assess the role of IL-33 in tumor growth in vivo. First, we established a subcutaneous syngeneic mouse model. Similar tumor growth rates were observed between the ID8-mock and ID8-IL33 cells in this model (Fig. 1E).
The volumes calculated in Fig. 1E were for one tumor per mouse. We then established a syngeneic mouse model of peritoneal carcinomatosis and compared their survival. The survival rate of the ID8-IL33 tumor-bearing mice was not shortened, but was significantly prolonged compared with that of the ID8-mock tumor-bearing mice ( $\mathrm{P}=0.001$; Fig. 1F). The effects of IL-33 on tumors is controversial, and there are some reports stating that IL-33 enhances antitumor immunity and suppresses tumor growth (27-29).

Considering the possibility that IL-33 acts on tumor immunity, we examined the localization of immune cells (CD4, CD8, CD11b and F4/80) within the disseminated tumor tissues by IHC analyses (Fig. 1G). The numbers of infiltrating $\mathrm{CD}^{+}$cells and $\mathrm{CD}^{+}$cells were significantly increased in the ID8-IL33 tumors compared with the ID8-mock tumors. By contrast, the numbers of infiltrating CD11b ${ }^{+}$cells were decreased in ID8-IL33 tumors. No marked no difference was observed in the numbers of infiltrating $\mathrm{F} 4 / 80^{+}$cells. These results suggested that IL-33 inhibits tumor growth by promoting antitumor immunity.

IL-33 in ascites directly inhibits myeloid-derived cell differentiation. IL-33 increased the number of infiltrating $\mathrm{CD}^{+}$and $\mathrm{CD}^{+}$cells, and decreased the amount of infiltrating $\mathrm{CD}_{11} \mathrm{~b}^{+}$cells. Based on these results, we considered the involvement of myeloid-derived suppressor cells (MDSCs). MDSCs are characterized as $\mathrm{CD}_{1} \mathrm{~b}^{+} \mathrm{Gr}-1^{+}$cells in mice. In cancer, MDSCs have a prominent immunosuppressive ability that enables them to control immune responses, leading to tumor immune escape and disease progression (30). Therefore, in this study, we examined intratumoral $\mathrm{CD} 11 \mathrm{~b}^{+} \mathrm{Gr}-1^{+}$cells by FCM. The number of intratumoral $\mathrm{CD} 11 \mathrm{~b}^{+} \mathrm{Gr}-1^{+}$cells was significantly decreased in the IL33-overexpressing tumors 
A

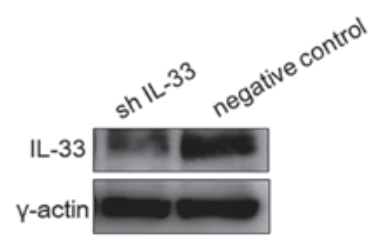

C

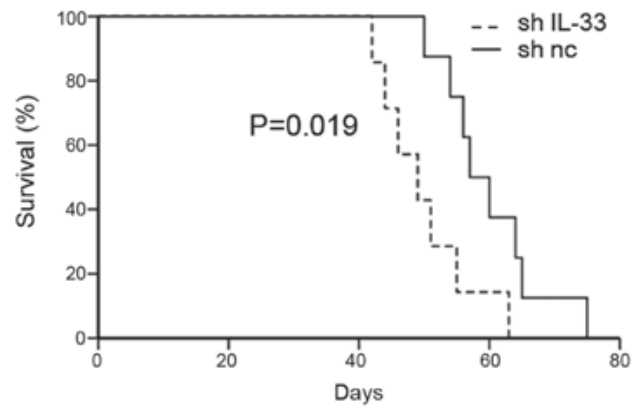

D

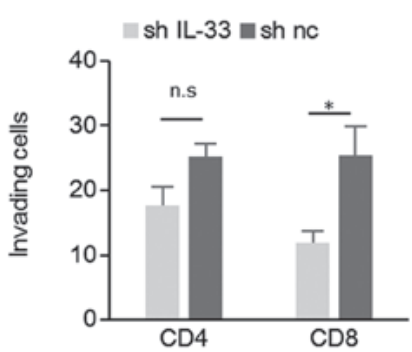

B

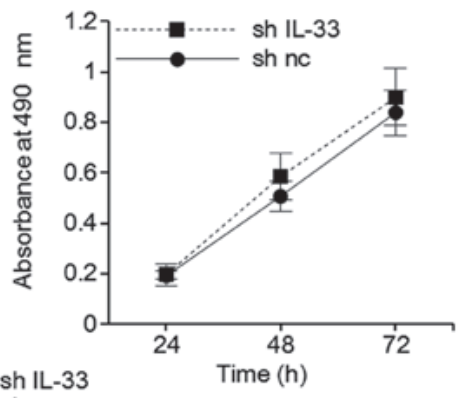

$\mathbf{E}$

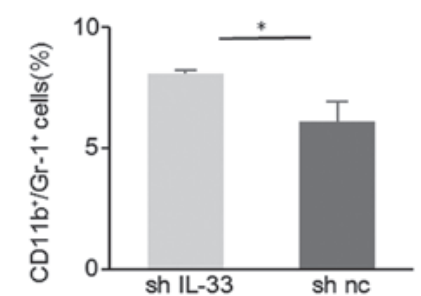

Figure 3. Silencing of IL-33 suppresses tumor immunity and promotes tumor progression. (A) Western blot analysis of IL33 expression in shIL-33 and sh negative control cells. (B) Cell proliferation was measured over a period of 3 days in shIL-33 and sh negative control cells by MTT assay. No difference in proliferation was observed. (C) Overall survival rates were compared between mice intraperitoneally injected with shIL-33 ( $\mathrm{n}=7)$ or sh negative control $(\mathrm{n}=8)$ cells $\left(5 \times 10^{6}\right)$. (D) Analyses of the infiltration of $\mathrm{CD}^{+}$and $\mathrm{CD}^{+}$cells within shIL-33 $(\mathrm{n}=4)$ or sh negative control $(\mathrm{n}=4)$ tumors by IHC. (E) The percentage of $\mathrm{CD}_{11} \mathrm{~b}^{+} \mathrm{Gr} 1^{+}$cells in shIL-33 $(\mathrm{n}=5)$ or sh negative control $(\mathrm{n}=5)$ tumor cells by flow cytometry. ${ }^{*} \mathrm{P}<0.05 ; \mathrm{n} . \mathrm{s}$, not significant.

(Fig. 2A). This suggested that immune activation was also mediated through MDSC inhibition, although it was likely due to the direct effects of IL-33 on lymphocytes. No difference was observed in tumor growth in the subcutaneous model; but a difference was observed in the model of peritoneal dissemination. Thus, we considered that cancerous ascites may be involved in the reduction of intratumoral $\mathrm{CD}_{11 \mathrm{~b}} \mathrm{Gr}^{+} \mathrm{1}^{+}$ cells, as ovarian cancer ascitic fluids contain several cytokines, such as IL-6, IL-10 and GM-CSF, which induce differentiation into MDSCs (31-33). To investigate the effects of cancerous ascites on MDSCs, we collected bone marrow cells from the femurs of healthy $\mathrm{C} 57 \mathrm{Bl} / 6$ mice and incubated them with ascites from ID8-IL33 and ID8-mock tumor-bearing mice. The number of $\mathrm{CD} 11 \mathrm{~b}^{+} \mathrm{Gr}-1^{+}$cells induced by ascites from the ID8-IL33 mice was significantly lower than that induced by ascites from ID8-mock mice (Fig. 2B). Ascites from the ID8-IL33 tumor-bearing mice inhibited myeloid-derived cell differentiation. Subsequently, we investigated what factor in the ascites acts to prevent differentiation into $\mathrm{CD}_{11} \mathrm{~b}^{+} \mathrm{Gr}-1^{+}$ cells. First, the concentration of IL-33 in the mouse ascites was measured by ELISA. The IL-33 concentration in ascites from the ID8-IL33 tumor-bearing mice was significantly higher, whereas ascites from ID8-mock tumor-bearing mice were almost free of IL-33 (Fig. 2C). IL-33-responsive innate cells have been previously reported in mouse bone marrow (34). Therefore, we considered that IL-33 in ascites may directly inhibit the differentiation of lineage-negative progenitor bone marrow cells into $\mathrm{CD}_{1} 1 \mathrm{~b}^{+} \mathrm{Gr}-1^{+}$cells. We added recombinant IL-33 to IL-33-free ascites from ID8-mock tumor-bearing mice and cultured with bone marrow cells from healthy C57BL/6 mice. The addition of recombinant IL-33 reduced myeloid-derived cell differentiation. However, the effect plateaued when the rIL33 concentration reached a certain level (Fig. 2D). These results suggested that IL-33 in ascites inhibited MDSC differentiation induced by cytokines, thereby promoting tumor immunity.

Silencing of IL-33 suppresses tumor immunity and promotes tumor progression. Although IL-33 was found to be overexpressed in ID8-T6 cells, ID8-T6 significantly shortened the survival of mice in a murine model of peritoneal carcinomatosis compared with ID8-WT (Fig. S2B). To clarify this contradiction, we generated IL33-a-silenced ID8-T6 cells, shIL-33 and a negative control. The knockdown of IL-33 in the shIL-33 cells was confirmed by WB analysis (Fig. 3A). Cell proliferation in vitro was similar between the shIL-33 and negative control-transfected cells (Fig. 3B). In a murine model of peritoneal carcinomatosis, the shIL-33 tumor-bearing mice exhibited significantly shorter survival times $(\mathrm{P}=0.019$; Fig. 3C). Furthermore, the silencing of IL-33 reduced the number of infiltrating $\mathrm{CD} 8^{+}$cells, and increased the number of $\mathrm{CD} 11 \mathrm{~b}^{+} \mathrm{Gr}-1^{+}$cells (Fig. 3D and E). This result suggested 
A
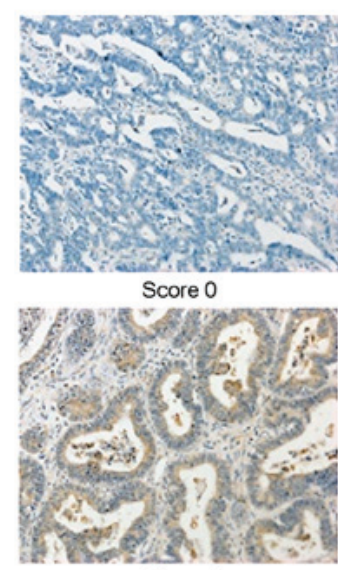

Score 2

C
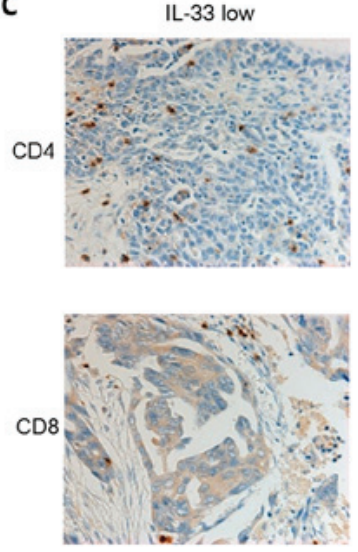
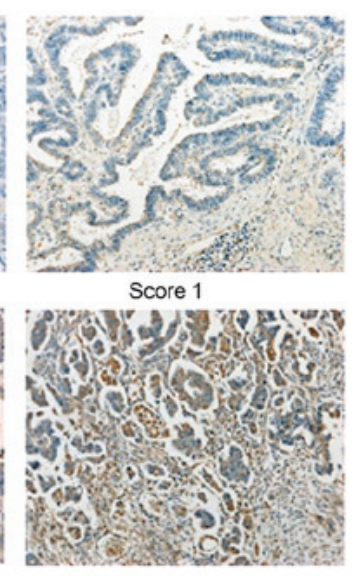

Score 3

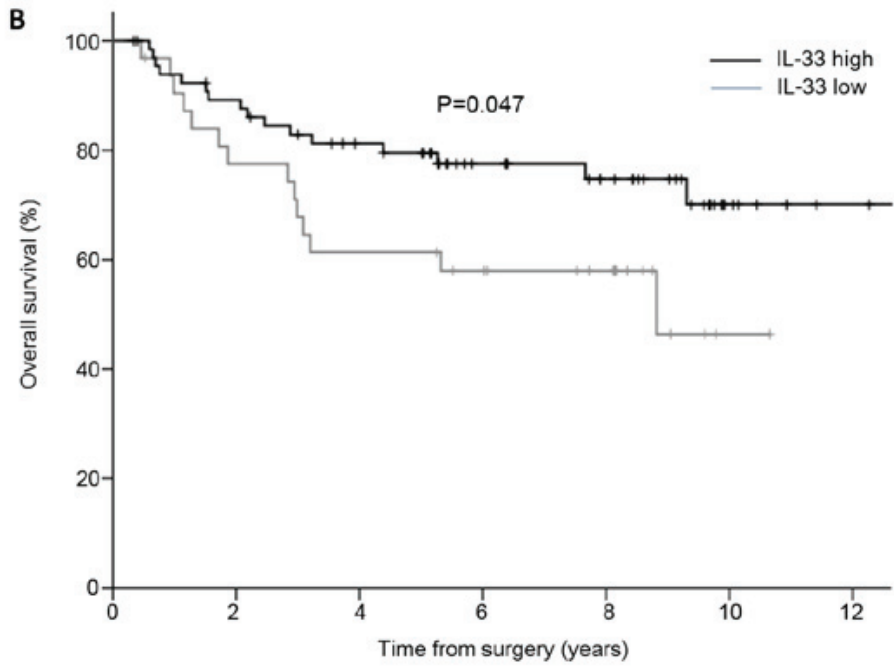

Figure 4. Association of IL-33 with clinical outcome in human ovarian cancer. (A) Classification of ovarian cancer according to the intensity of IL-33 staining. Cases with scores of 0, 1 were assigned to the IL-33 low group and cases with scores of 2, 3 were assigned to the IL-33 high group. (B) Survival analyses for patients with ovarian cancer according to intensity of IL-33. Overall survival rates were compared between the IL-33 low group and IL-33 high group. (C) Representative immunostaining images of IL-33 low or high tumors for CD4 and CD8. Analyses of the infiltration of CD4 ${ }^{+}$and CD8 ${ }^{+}$cells within IL-33 low or high tumors. ${ }^{*} \mathrm{P}<0.05$; n.s, not significant.

that IL-33 also suppressed tumor progression through tumor immunity in ID8-T6 cells and confirmed the antitumor effects of IL-33.

Association of IL-33 with clinical outcome in human ovarian cancer. Taken together, our preclinical results demonstrated that IL-33 improves prognosis by enhancing tumor immunity. To assess whether this finding is clinically relevant, we examined the expression of IL-33 in 100 paraffin-fixed human primary ovarian cancer tissues (Table I). We found different expression levels of IL-33 in ovarian cancer cells. We classified scores 0/1 as IL-33 low and 2/3 as IL-33 high. In total, 67 of the samples expressed high levels of IL-33 (IL-33 high) in the ovarian cancer cells (Table I and Fig. 4A). Cases with high staining of IL-33 had significantly longer overall survival times ( $\mathrm{P}=0.047$; Fig. 4B). To confirm the association of tumor IL-33 expression with antitumor immunity, we performed additional IHC staining for CD4 and CD8. Although no differences were observed in the number of infiltrating $\mathrm{CD}^{+}$ cells, the numbers of infiltrating $\mathrm{CD} 8^{+}$cells were significantly increased in the IL-33 high expression group (Fig. 4C). These findings suggest that IL-33 enhances tumor immunity and prolongs survival.

\section{Discussion}

The importance of interactions in both cancer and tumor microenvironments for the malignant phenotype and genotype of EOC has been demonstrated in recent studies (35-37). These complex interactions may be due to the accumulation of spatiotemporal signaling events that function in tumor progression or regression. Due to the heterogeneity of EOC, the underlying mechanisms altering gene expression remain unclear. These alterations involve a number of secreted components, such as cytokines, chemokines, growth factors, metabolites and exosomal miRNAs (38). These non-cellular components under peritoneal carcinomatosis conditions may have a greater influence on the acquisition of malignancy by providing energy, growth signals, drug resistance tumor microenvironment, evasion of immune surveillance, and metastatic and angiogenesis cues. Peritoneal carcinomatosis is a devastating metastatic form of EOC. Thus, switching the function of these secreted components from tumor acceleration to antitumor activity has been considered as a new target for EOC therapy.

Based on this, we searched for potential factors involved in peritoneal carcinomatosis. We focused on IL-33, which is a humoral factor that has recently been reported to be 
associated with cancer. We initially hypothesized that IL-33 promotes tumorigenesis; however, the overexpression of IL-33 significantly prolonged the survival of mice in a murine model of peritoneal carcinomatosis. By contrast, the knockdown of IL-33 in ID8-T6 cells significantly shortened the survival of mice. Thus, IL-33 enhanced tumor immunity and suppressed tumor growth in a murine model of peritoneal carcinomatosis. As to why ID8-T6 acquired a high dissemination ability, several progression factors that have a greater influence on peritoneal dissemination than IL-33 are likely to be expressed in ID8-T6 cells. Thus, the antitumor effects of IL-33 are likely subverted due to interactions with other tumor promoting factors. Accordingly, we intraperitoneally injected ID8-WT or ID8-T6 cells into immunodeficient mice (BALB/C nude). Similar as in immunocompetent mice, peritoneal dissemination formation by ID8-T6 cells was earlier than that by ID8-WT cells (data not shown). We considered a number of potent progression factors, regardless of immunity, to be expressed in ID8-T6 cells, and that the antitumor effects of IL-33 were masked.

Although IL-33 was highly expressed in ID8-T6 cells, IL-33 is a naturally acquired tumor suppressor in the process of ID8 with recurrent peritoneal dissemination. Therefore, the expression of IL-33 in the tumor may finally increase as the tumor progresses to a certain extent in humans. Indeed, it was previously reported that the expression levels of IL-33 were further increased in tumor tissues at the metastatic site compared with at the primary site (23). It may therefore be difficult to use IL-33 as a prognostic marker, even though it significantly prolonged human survival in this study.

In this study, we demonstrated that IL-33 overexpression promoted the infiltration of $\mathrm{CD}^{+}$and $\mathrm{CD}^{+}$cells into tumors in a syngeneic mouse model of peritoneal carcinomatosis. Infiltrating $\mathrm{CD}^{+}$cells isolated from the disseminated tumors have the ability of functional cytokine production. Although tumor-infiltrating $\mathrm{CD}^{+} \mathrm{T}$ cell function was similar between ID8-mock and ID8-IL33 peritoneal tumor settings, the number of infiltrating $\mathrm{CD}^{+}$cells differed (data not shown).

Furthermore, in 100 clinical cases of ovarian cancer, CD8 ${ }^{+}$ cells were significantly increased in the IL-33 high group. The expression of ST2 was detected on Th2 cells $(39,40)$. Recently, activated Th1 cells (41) and cytotoxic T cells (42) were found to have ST2 expression and IL-33 signaling. Thus, it is possible that IL-33 directly acts on $\mathrm{CD} 4^{+}$and $\mathrm{CD}^{+}$cells to enhance tumor immunity. However, as a significant difference was observed in the number of $\mathrm{CD} 11 \mathrm{~b}^{+}$cells, as opposed to $\mathrm{CD}^{+}$or $\mathrm{CD}^{+}$cells, in this study, we hypothesized that some other signaling mechanism is involved. Tumor-derived cytokines and inflammatory cytokines, including VEGF, prostaglandin E2 (PGE2), TGF- $\beta$, stem cell factor (SCF), IL-1 $\beta$, IL-4, IL-6, IL-10, IL-12, IL-13, matrix metalloproteinase (MMP)-9, granulocyte-macrophage colony-stimulating factor (GM-CSF) and granulocyte-colony stimulating factor (G-CSF), have been reported to play a role in MDSC differentiation $(43,44)$. Furthermore, it was recently reported that IL-33 effectively reduces MDSC-dependent immune suppression and improves antitumor responses (45). Therefore, we assumed that the increase in infiltrating $\mathrm{CD}^{+}$and $\mathrm{CD}^{+}$ cells was mediated by MDSCs. Indeed, we demonstrated that IL-33 in cancerous ascites was able to inhibit the differentiation of bone marrow cells into $\mathrm{CD} 11 \mathrm{~b}^{+} \mathrm{Gr}-1^{+}$cells. Although we used CD11b and Gr-1 as markers to identify MDSC, these markers are also expressed by other cells. Indeed, the only way to definitively identify cells as MDSCs is to demonstrate that they have immune suppressive activity. However, there was a limitation in our technical ability to evaluate the $\mathrm{T}$ cell-suppressive activity of tumor infiltrating $\mathrm{CD} 11 \mathrm{~b}^{+} \mathrm{Gr}-1^{+}$ cells, as we were unable to use the FACS cell sorter to purify CD11b/Gr-1 double-positive cells. It is possible that other mechanisms are involved and influenced the survival, but it is certain that this mechanism is involved.

In this study, no significant difference was observed in tumor growth by the overexpression of IL-33 in cancer cells in a subcutaneous syngeneic mouse model, whereas the overexpression of IL-33 significantly prolonged the survival of mice in a murine model of peritoneal carcinomatosis. We considered this to be a unique result from peritoneal dissemination. IL-33 expressed by the tumor acts only locally in the subcutaneous model; however, IL-33 expressed by tumor cells may act extensively via cancerous ascites in a murine model of peritoneal carcinomatosis. Even in hepatocellular carcinoma that is likely to develop peritoneal carcinomatosis, such as ovarian cancer, the infiltration of cancer cells by IL-33 ${ }^{+}$ and $\mathrm{CD}^{+}$cells is independently associated with prolonged patient survival (46). In the experiment using IL-33 transgenic mice, IL-33 promoted the proliferation, activation and infiltration of $\mathrm{CD}^{+} \mathrm{T}$ cells, and the inhibition of pulmonary metastasis in the B16 melanoma and Lewis lung carcinoma metastatic mice models (28). It is believed that IL-33 enhances tumor immunity and exhibits antitumor effects only when it functions systemically.

In conclusion, we demonstrated that IL-33 reduces tumor immune suppression and inhibits tumor progression in ovarian cancer. Furthermore, we suggest that the reduction of MDSCs by IL-33 reduced immune suppression. Further the elucidation of the antitumorigenic role of IL-33 will aid in developing novel therapeutic approaches for the treatment of EOC with peritoneal carcinomatosis in the future.

\section{Acknowledgements}

Not applicable.

\section{Funding}

This study was supported by JSPS KAKENHI (grant no. 15K20138) (research funding).

\section{Availability of data and materials}

The datasets used and/or analyzed during the current study are available from the corresponding author upon reasonable request.

\section{Authors' contributions}

AS and SS conceived the study, analyzed and interpreted data, performed statistical analyses and wrote the manuscript. AT, $\mathrm{SH}$ and YS participated in data collection and the provision of patient samples. NY, YK and HK aided in the design of the study, and collected and assembled the data. FK was involved in the conception and design of the study, coordinated the study over 
the entire period and participated in editing and proofreading. All authors have read and approved the final manuscript.

\section{Ethics approval and consent to participate.}

This study was approved by the Institutional Review Board at Nagoya University School of Medicine (approval no. for the use of human samples: 2017-0053). Informed consent was obtained in the form of opt-out on the web-site (https://www. med.nagoya-u.ac.jp/medical_J/ethics/pdf/1024_2017-0053.pdf). Those who rejected were excluded. Animal care and experimental procedures were approved by the Animal Experiment Committee of Nagoya University (approval no. 30065).

\section{Patient consent for publication}

Not applicable.

\section{Competing interests}

The authors declare that they have no competing interests.

\section{References}

1. Ebell MH, Culp MB and Radke TJ: A Systematic review of symptoms for the diagnosis of ovarian cancer. Am J Prev Med 50: 384-394, 2016.

2. Coleman RL, Monk BJ, Sood AK and Herzog TJ: Latest research and treatment of advanced-stage epithelial ovarian cancer. Nat Rev Clin Oncol 10: 211-224, 2013.

3. Chae CS, Teran-Cabanillas E and Cubillos-Ruiz JR: Dendritic cell rehab: New strategies to unleash therapeutic immunity in ovarian cancer. Cancer Immunol Immunother 66: 969-977, 2017.

4. Suzuki S, Sakata J, Utsumi F, Sekiya R, Kajiyama H, Shibata K, Kikkawa F and Nakatsura T: Efficacy of glypican-3-derived peptide vaccine therapy on the survival of patients with refractory ovarian clear cell carcinoma. OncoImmunology 5: e1238542, 2016.

5. Zhu X, Cai H, Zhao L, Ning L and Lang J: CAR-T cell therapy in ovarian cancer: From the bench to the bedside. Oncotarget 8 : 64607-64621, 2017.

6. Haraldsen G, Balogh J, Pollheimer J, Sponheim J and Küchler AM: Interleukin-33 - cytokine of dual function or novel alarmin? Trends Immunol 30: 227-233, 2009.

7. Liew FY, Pitman NI and McInnes IB: Disease-associated functions of IL-33: The new kid in the IL-1 family. Nat Rev Immunol 10: 103-110, 2010.

8. Schmitz J, Owyang A, Oldham E, Song Y, Murphy E, McClanahan TK, Zurawski G, Moshrefi M, Qin J, Li X, et al: IL-33, an interleukin-1-like cytokine that signals via the IL-1 receptor-related protein ST2 and induces T helper type 2-associated cytokines. Immunity 23: 479-490, 2005.

9. Oboki K, Ohno T, Kajiwara N, Saito H and Nakae S: IL-33 and IL-33 receptors in host defense and diseases. Allergol Int 59: $143-160,2010$

10. Sattler S, Smits HH, Xu D and Huang FP: The evolutionary role of the IL-33/ST2 system in host immune defence. Arch Immunol Ther Exp (Warsz) 61: 107-117, 2013.

11. Kurowska-Stolarska M, Kewin P, Murphy G, Russo RC, Stolarski B, Garcia CC, Komai-Koma M, Pitman N, Li Y, Niedbala W, et al: IL-33 induces antigen-specific IL- $5^{+} \mathrm{T}$ cells and promotes allergic-induced airway inflammation independent of IL-4. J Immunol 181: 4780-4790, 2008.

12. Kurowska-Stolarska M, Stolarski B, Kewin P, Murphy G, Corrigan CJ, Ying S, Pitman N, Mirchandani A, Rana B, van Rooijen N, et al: IL-33 amplifies the polarization of alternatively activated macrophages that contribute to airway inflammation. J Immunol 183: 6469-6477, 2009.

13. Pei C, Barbour M, Fairlie-Clarke KJ, Allan D, Mu R and Jiang HR: Emerging role of interleukin-33 in autoimmune diseases. Immunology 141: 9-17, 2014.
14. Wang S, Ding L, Liu SS, Wang C, Leng RX, Chen GM, Fan YG, Pan HF and Ye DQ: IL-33: A potential therapeutic target in autoimmune diseases. J Investig Med 60: 1151-1156, 2012.

15. Miller AM, Xu D, Asquith DL, Denby L, Li Y, Sattar N, Baker AH, McInnes IB and Liew FY: IL-33 reduces the development of atherosclerosis. J Exp Med 205: 339-346, 2008.

16. Sanada S, Hakuno D, Higgins LJ, Schreiter ER, McKenzie AN and Lee RT: IL-33 and ST2 comprise a critical biomechanically induced and cardioprotective signaling system. J Clin Invest 117: 1538-1549, 2007.

17. Yu XX, Hu Z, Shen X, Dong LY, Zhou WZ and Hu WH: IL-33 promotes gastric cancer cell invasion and migration via ST2-ERK1/2 pathway. Dig Dis Sci 60: 1265-1272, 2015.

18. Zhang P, Liu XK, Chu Z, Ye JC, Li KL, Zhuang WL, Yang DJ and Jiang YF: Detection of interleukin-33 in serum and carcinoma tissue from patients with hepatocellular carcinoma and its clinical implications. J Int Med Res 40: 1654-1661, 2012.

19. Kim JY, Lim SC, Kim G, Yun HJ, Ahn SG and Choi HS: Interleukin-33/ST2 axis promotes epithelial cell transformation and breast tumorigenesis via upregulation of COT activity. Oncogene 34: 4928-4938, 2015.

20. Liu J, Shen JX, Hu JL, Huang WH and Zhang GJ: Significance of interleukin-33 and its related cytokines in patients with breast cancers. Front Immunol 5: 141, 2014

21. O'Donnell C, Mahmoud A, Keane J, Murphy C, White D, Carey S, O'Riordain M, Bennett MW, Brint E and Houston A: An antitumorigenic role for the IL-33 receptor, ST2L, in colon cancer. Br J Cancer 114: 37-43, 2016.

22. Akimoto M, Hayashi JI, Nakae S, Saito H and Takenaga K: Interleukin-33 enhances programmed oncosis of ST2L-positive low-metastatic cells in the tumour microenvironment of lung cancer. Cell Death Dis 7: e2057, 2016.

23. Tong X, Barbour M, Hou K, Gao C, Cao S, Zheng J, Zhao Y, Mu R and Jiang HR: Interleukin-33 predicts poor prognosis and promotes ovarian cancer cell growth and metastasis through regulating ERK and JNK signaling pathways. Mol Oncol 10: 113-125, 2016.

24. Saied EM and El-Etreby NM: The role and prognostic value of inducible nitric oxide synthase (iNOS) and interleukin-33 (IL-33) in serous and mucinous epithelial ovarian tumours. Ann Diagn Pathol 27: 62-68, 2017.

25. Livak KJ and Schmittgen TD: Analysis of relative gene expression data using real-time quantitative PCR and the 2(- $\Delta \Delta \mathrm{C}(\mathrm{T}))$ Method. Methods 25: 402-408, 2001.

26. Suzuki S, Terauchi M, Umezu T, Kajiyama H, Shibata K, Nawa A and Kikkawa F: Identification and characterization of cancer stem cells in ovarian yolk sac tumors. Cancer Sci 101: 2179-2185, 2010.

27. Villarreal DO, Wise MC, Walters JN, Reuschel EL, Choi MJ, Obeng-Adjei N, Yan J, Morrow MP and Weiner DB: Alarmin IL-33 acts as an immunoadjuvant to enhance antigen-specific tumor immunity. Cancer Res 74: 1789-1800, 2014.

28. Gao K, Li X, Zhang L, Bai L, Dong W, Gao K, Shi G, Xia X, Wu L and Zhang L: Transgenic expression of IL-33 activates CD8(+) T cells and NK cells and inhibits tumor growth and metastasis in mice. Cancer Lett 335: 463-471, 2013.

29. Gao X, Wang X, Yang Q, Zhao X, Wen W, Li G, Lu J, Qin W, Qi Y, Xie F, et al: Tumoral expression of IL-33 inhibits tumor growth and modifies the tumor microenvironment through $\mathrm{CD}^{+} \mathrm{T}$ and NK cells. J Immunol 194: 438-445, 2015.

30. Millrud CR, Bergenfelz C and Leandersson K: On the origin of myeloid-derived suppressor cells. Oncotarget 8: 3649-3665, 2017.

31. Kolomeyevskaya N, Eng KH, Khan AN, Grzankowski KS, Singel KL, Moysich K and Segal BH: Cytokine profiling of ascites at primary surgery identifies an interaction of tumor necrosis factor- $\alpha$ and interleukin- 6 in predicting reduced progression-free survival in epithelial ovarian cancer. Gynecol Oncol 138: 352-357, 2015.

32. Morales JK, Kmieciak M, Knutson KL, Bear HD and Manjili MH: GM-CSF is one of the main breast tumor-derived soluble factors involved in the differentiation of $\mathrm{CD}_{11} \mathrm{~b}^{-} \mathrm{Grl}^{-}$bone marrow progenitor cells into myeloid-derived suppressor cells. Breast Cancer Res Treat 123: 39-49, 2010.

33. Wu L, Deng Z, Peng Y, Han L, Liu J, Wang L, Li B, Zhao J, Jiao $S$ and Wei H: Ascites-derived IL-6 and IL-10 synergistically expand $\mathrm{CD} 14^{+} \mathrm{HLA}-\mathrm{DR}^{-/ \mathrm{low}}$ myeloid-derived suppressor cells in ovarian cancer patients. Oncotarget 8: 76843-76856, 2017.

34. Brickshawana A, Shapiro VS, Kita H and Pease LR: Lineage(-) $\mathrm{Scal}^{+} \mathrm{c}-\mathrm{Kit}(-) \mathrm{CD} 25^{+}$cells are IL-33-responsive type 2 innate cells in the mouse bone marrow. J Immunol 187: 5795-5804, 2011. 
35. Mitsui H, Shibata K, Suzuki S, Umezu T, Mizuno M, Kajiyama H and Kikkawa F: Functional interaction between peritoneal mesothelial cells and stem cells of ovarian yolk sac tumor (SC-OYST) in peritoneal dissemination. Gynecol Oncol 124: 303-310, 2012.

36. Lau TS, Chan LK, Wong EC, Hui CW, Sneddon K, Cheung TH, Yim SF, Lee JH, Yeung CS, Chung TK, et al: A loop of cancer-stroma-cancer interaction promotes peritoneal metastasis of ovarian cancer via TNF $\alpha$-TGF $\alpha$-EGFR. Oncogene 36 : 3576-3587, 2017.

37. Fujikake K, Kajiyama H, Yoshihara M, Nishino K, Yoshikawa N Utsumi F, Suzuki S, Niimi K, Sakata J, Mitsui H, et al: A novel mechanism of neovascularization in peritoneal dissemination via cancer-associated mesothelial cells affected by TGF- $\beta$ derived from ovarian cancer. Oncol Rep 39: 193-200, 2018.

38. Yokoi A, Yoshioka Y, Yamamoto Y, Ishikawa M, Ikeda SI, Kato T, Kiyono T, Takeshita F, Kajiyama H, Kikkawa F, et al: Malignant extracellular vesicles carrying MMP1 mRNA facilitate peritoneal dissemination in ovarian cancer. Nat Commun 8: 14470, 2017.

39. Löhning M, Stroehmann A, Coyle AJ, Grogan JL, Lin S, Gutierrez-Ramos JC, Levinson D, Radbruch A and Kamradt T: T1/ST2 is preferentially expressed on murine Th2 cells, independent of interleukin 4, interleukin 5, and interleukin 10, and important for Th2 effector function. Proc Natl Acad Sci USA 95: 6930-6935, 1998.

40. Xu D, Chan WL, Leung BP, Huang F, Wheeler R, Piedrafita D, Robinson JH and Liew FY: Selective expression of a stable cell surface molecule on type 2 but not type 1 helper T cells. J Exp Med 187: 787-794, 1998.
41. Baumann C, Bonilla WV, Fröhlich A, Helmstetter C, Peine M, Hegazy AN, Pinschewer DD and Löhning M: T-bet- and STAT4-dependent IL-33 receptor expression directly promotes antiviral Th1 cell responses. Proc Natl Acad Sci USA 112: 4056-4061, 2015.

42. Bonilla WV, Fröhlich A, Senn K, Kallert S, Fernandez M, Johnson S, Kreutzfeldt M, Hegazy AN, Schrick C, Fallon PG, et al: The alarmin interleukin-33 drives protective antiviral CD $8^{+} \mathrm{T}$ cell responses. Science 335: 984-989, 2012.

43. Gabrilovich DI and Nagaraj S: Myeloid-derived suppressor cells as regulators of the immune system. Nat Rev Immunol 9: 162-174, 2009.

44. Ribechini E, Greifenberg V, Sandwick S and Lutz MB: Subsets, expansion and activation of myeloid-derived suppressor cells. Med Microbiol Immunol (Berl) 199: 273-281, 2010.

45. Lim HX, Choi S, Cho D and Kim TS: IL-33 inhibits the differentiation and immunosuppressive activity of granulocytic myeloid-derived suppressor cells in tumor-bearing mice. Immunol Cell Biol 95: 99-107, 2017.

46. Brunner SM, Rubner C, Kesselring R, Martin M, GriesshammerE, Ruemmele P, Stempfl T, Teufel A, Schlitt HJ and Fichtner-Feigl S: Tumor-infiltrating, interleukin-33-producing effector-memory CD8(+) T cells in resected hepatocellular carcinoma prolong patient survival. Hepatology 61: 1957-1967, 2015. 